\title{
Analítica de datos en simulador de redes para sistemas de medición inteligente
}

\author{
Juan C. Olivares-Rojas ${ }^{1}$, Enrique Reyes-Archundia ${ }^{1}$, José A. Gutiérrez-Gnecchi ${ }^{1}$, \\ Johan W. González-Murueta ${ }^{1}$, Adriana Téllez-Anguiano ${ }^{1}$, Jaime Cerda-Jacobo ${ }^{2}$ \\ ${ }^{1}$ Tecnológico Nacional de México / Instituto Tecnológico de Morelia, \\ Morelia, Michoacán, México \\ ${ }^{2}$ Universidad Michoacana de San Nicolás de Hidalgo, Facultad de Ingeniería Eléctrica, \\ Morelia, Michoacán, México \\ \{jcolivares, ereyes, agnecchi, jwalter, \\ adrianat\}@itmorelia.edu.mx, jcerda@umich.mx
}

\begin{abstract}
Resumen. La Red Eléctrica Inteligente es uno de los pilares fundamentales de la Transformación Digital producida por la cuarta revolución industrial. Muchos de los problemas de la red eléctrica inteligente como los sistemas de medición inteligente no pueden modelarse a través de pruebas reales de laboratorio debido a la densidad de componentes utilizados; para ello, es necesario utilizar simuladores de redes eléctricas. La gran mayoría de los simuladores de redes eléctricas no cuentan con muchas capacidades de comunicación a redes de datos ni permiten realizar analítica de datos. Este trabajo presenta la adecuación del simulador de redes PacketTracer para permitir el manejo de analítica de datos en sistemas de medición inteligente.
\end{abstract}

Palabras clave: analítica de datos, simulador de redes, medidor inteligente, sistema de medición inteligente.

\section{Data Analytic in Networks Simulator for Smart Metering Systems}

\begin{abstract}
The Smart Grid is one of the fundamental pillars of the Digital Transformation produced by the fourth industrial revolution. Many of the problems of the smart grid such as smart metering systems cannot be modeled through real laboratory tests due to the density of components used; for this, it is necessary to use simulators of electrical networks. The vast majority of electrical network simulators do not have many communication capabilities to data networks or allow data analytics. This paper presents the suitability of the PacketTracer network simulator to allow the management of data analytics in smart metering systems.
\end{abstract}

Keywords: data analytics, network simulator, smart meter, smart metering system. 


\section{Introducción}

La red eléctrica constituye un sistema sumamente complejo e interconectado. En los últimos años se han agregado Tecnologías de Información y Comunicaciones (TICs) a la red eléctrica dándole capacidades de comunicación y procesamiento de datos [1]. Esto a llevado a darle algún grado de inteligencia a la red eléctrica tradicional, motivo por el cual a pasado a denominarse Red Eléctrica Inteligente (REI).

Para los usuarios finales, una de las aplicaciones más visible de la REI, son los Sistemas de Medición Inteligente (SMI) caracterizados por el reporte periódico de los datos de consumo por parte de los medidores de energía eléctrica hacia los centros de datos de las empresas eléctricas [2]. Para ello, el medidor ha sido dotado de capacidades de comunicación de datos a través de redes cableadas e inalámbricas, motivo por el cual ha recibido el nombre de medidor inteligente.

Los medidores inteligentes, son dispositivos empotrados que cuentan con una pequeña base de datos donde se guardan las lecturas de las mediciones de energía eléctrica. Recientemente, con la integración de los recursos de energía distribuidos principalmente de fuentes renovables como la fotovoltaica y eólica, los medidores inteligentes han integrado capacidades para medir la producción de energía eléctrica [1].

La implementación mejor conocida de los SMI es la infraestructura de medición avanzada (AMI, por sus siglas en inglés). Los sistemas AMI permiten además del monitoreo de consumo/producción de energía en tiempo casi real de algunas otras funcionales como cortes y reconexiones de forma automática, señalización de precios dinámicos, así como la automatización de ahorro energético y respuesta a la demanda de los dispositivos conectados a la REI [2].

Todas las funcionalidades existentes en AMI y de las próximas en inventarse, están generando una gran cantidad de datos que necesitan ser procesados y analizados para la toma eficiente de decisiones energéticas [3].

Muchos de los problemas existentes en la REI no pueden ser modelados y probados de forma física debido a diversas cuestiones como los grandes voltajes, así como a no disponer de gran cantidad de componentes debido en muchos casos a los altos costos de los mismos. Por este motivo, los simuladores de redes son altamente utilizados.

La mayoría de los simuladores de redes existentes no cuentan con muchas capacidades de integración con las TICs, por lo que algunas aplicaciones como comunicación de datos, ciberseguridad, pero sobre todo analítica de datos, no están disponibles o lo están de forma parcial.

El presente artículo muestra una revisión de algunos simuladores de red eléctrica inteligente que existen, así como la adecuación de uno de ellos para modelar problemas de analítica de datos en SMI.

\section{Simuladores de redes eléctricas}

Existen diversos simuladores para el manejo de redes eléctricas en temas referentes a potencia en transmisión, distribución, generación y pocos se enfocan a la parte de consumo en particular en la parte de medición inteligente. 
La gran mayoría de estos simuladores son comerciales y de altos costos; y aunque la gran mayoría ofrecen versiones de prueba o licencias para uso académico, son generalmente más ligeras y con menos funcionalidades.

Algunos de los simuladores eléctricos más utilizados son CYME [4] de la empresa Cooper Power Systems, PowerFactory [5] de la empresa Digsilent, Neplan Smarter Tools [6], DSATools [7] de PowerTech Labs, etap Powering Success [8], SKM PowerTools [9], PowerWorld Simulator [10] de PowerWorld Corporation, EasyPower [11], entre otras. Estos simuladores carecen de buenas capacidades de comunicación y analítica de datos.

Por otra parte, otros de los simuladores existentes están enfocados en el manejo de circuitos eléctricos y el cálculo de valores. Entre los más destacados sobresalen: PSCAD/EMTC [12] de Manitoba Hydro International, Orcad [13], CircuitLab [14], entre otros. Dichos simuladores tampoco cuentan con buenas capacidades de comunicación y de análisis de datos.

Existen algunos entornos como Matlab [15] de la empresa MathWorks que permiten realizar simulaciones (a través de simulink [16]), los cuales se enfocan a diversas funcionales de simulación pero que cuentan con bibliotecas y componentes para realizar simulaciones en el campo eléctrico y de comunicaciones (Communication Systems Toolbox Support Package for RTL-SDR Radio [17], IoT [18], Comunicaciones Inalámbricas [19], Procesamiento Digital de Señales [20]).

En el ámbito de investigación se ha utilizado Matlab y otros simuladores de procesos tradicionales para simular el comportamiento de redes eléctricas inteligentes [21] y [22], con muy buenos resultados. De manera particular la comunicación y procesamiento de los datos se realizan de buena forma, aunque el modelar SMI aun no es posible de manera directa.

Además, existen simuladores que incluyen componentes de hardware especializado como RTDS [23], el cual permite simular no solo componentes de la red eléctrica sino aplicaciones como electrónica de potencia, micro redes, pruebas a sistemas de protecciones, Power Hardware In the Loop (PHIL) y pruebas de ciberseguridad. Otro buen simulador dentro de esta misma línea es HyperSIM de OPAL-RT [24] el cual incluye diversas aplicaciones de tiempo real no sólo para la industria eléctrica, en las últimas versiones incluyen elementos de ciberseguridad. Este producto puede integrarse con otros softwares como Matlab a través de la aplicación RT-LAB [25]. Esta clase de simuladores no cuentan con grandes capacidades de analítica de datos.

Por otra parte, existen simuladores de redes de datos como Cisco PacketTracer [26] enfocados al mundo de las telecomunicaciones y que recientemente han integrado elementos de Internet de las Cosas (IoT) entre los que se incluyen la REI. Desafortunadamente los simuladores de redes de computadoras no cuentan con una gran integración de componentes de la red eléctrica tradicional.

Como se ha podido observar en la presente sección, no existen como tales soluciones únicas que integren el mundo de la energía eléctrica, telecomunicaciones, sistemas embebidos, ciberseguridad y analítica de datos, entre otros componentes, para los problemas actuales de la REI y en particular SMI. Por este motivo, se han realizado en los últimos años diversos estudios comparativos y adecuaciones de simuladores [27, $28,29,30,31]$ para reflejar de mejor forma las condiciones actuales de la REI y SMI. 
También cabe destacar que año con año las actualizaciones de los principales simuladores de REI están incluyendo cada vez más y más capacidades de comunicación, ciberseguridad y analítica de datos.

\section{Simulador de Sistema de Medición Inteligente con Analítica de Datos}

Del análisis realizado en la sección anterior se notó que los dos mejores softwares de simulación para REI y SMI son Matlab y PacketTracer.

Debido a que la licencia de Matlab es de alto costo (y más si se agregan Toolboxes adicionales), así como a la experiencia previa con PacketTracer y a que no ha sido muy explotado por la comunidad de la Industria Eléctrica, se decidió explorar está herramienta, la cual es gratuita y sólo es necesario registrarse.

Además, PacketTracer presenta otras ventajas entre las que destacan que existe una gran variedad de dispositivos de IoT; las funciones de programación en IoT están basadas en Arduino. Aunque arduino está hecho en $\mathrm{C}$ se puede migrar fácilmente a JavaScript y Python que son los dos lenguajes base de este simulador. Adicionalmente se puede programar de forma visual a través de Blockly. Blockly es un lenguaje por bloques desarrollado por Google e implementado hoy en día en muchos Entornos de Desarrollo Integrados (IDEs). El lenguaje utilizado para la programación de las simulaciones fue Python debido a su versatilidad en el manejo de análisis de datos.

Aunque el simulador cuenta con varios componentes de interconexion a dispositivos de Internet de las Cosas, muy pocos son para REI. Por este motivo, se procedió a realizar una topología básica para simular un hogar inteligente, dado que la gran mayoría de componentes son para redes de área en el hogar (HAN por sus siglas en inglés).

La topología realizada se muestra en la Figura 1, la cual es una propuesta de un hogar inteligente, en donde existen distintos electrodomésticos cada uno de ellos con una pequeña base de datos empotrada, una placa Raspberry $\mathrm{Pi}$, y se conectan a la nube o una base de datos centralizada. Además se añadió un panel solar, así como un medidor inteligente y una batería de emergencia.

Cabe señalar que todos los dispositivos en la misma red local pueden intercomunicarse entre sí, o bien se puede controlar un dispositivo mediante un Smart Phone, Tableta o Computadora.

De manera predeterminada, todos los dispositivos de IoT del simulador disponen de una programación básica. Referente al consumo de energía eléctrica, el único dispositivo que tiene esta funcionalidad es la batería, mientras que de producción tanto el panel solar como el aerogenerador la poseen.

Debido a la limitante de consumo de energía se agregó una función de consumo de energía, para lo cual se tomaron valores de consumo de referencia. Por ejemplo, a la lámpara inteligente se le puso un valor de $60 \mathrm{w}$. Esta función es genérica y puede colocarse a cada tipo de dispositivo, midiendo su consumo por hora o fracción de hora. Los valores de consumo de energía pueden enviarse de forma automática al medidor inteligente o bien consultarse a través de un pequeño servicio Web desarrollado dentro del simulador. Esta funcionalidad de reporte de datos fue extendida a la batería, panel solar y aerogenerador. 


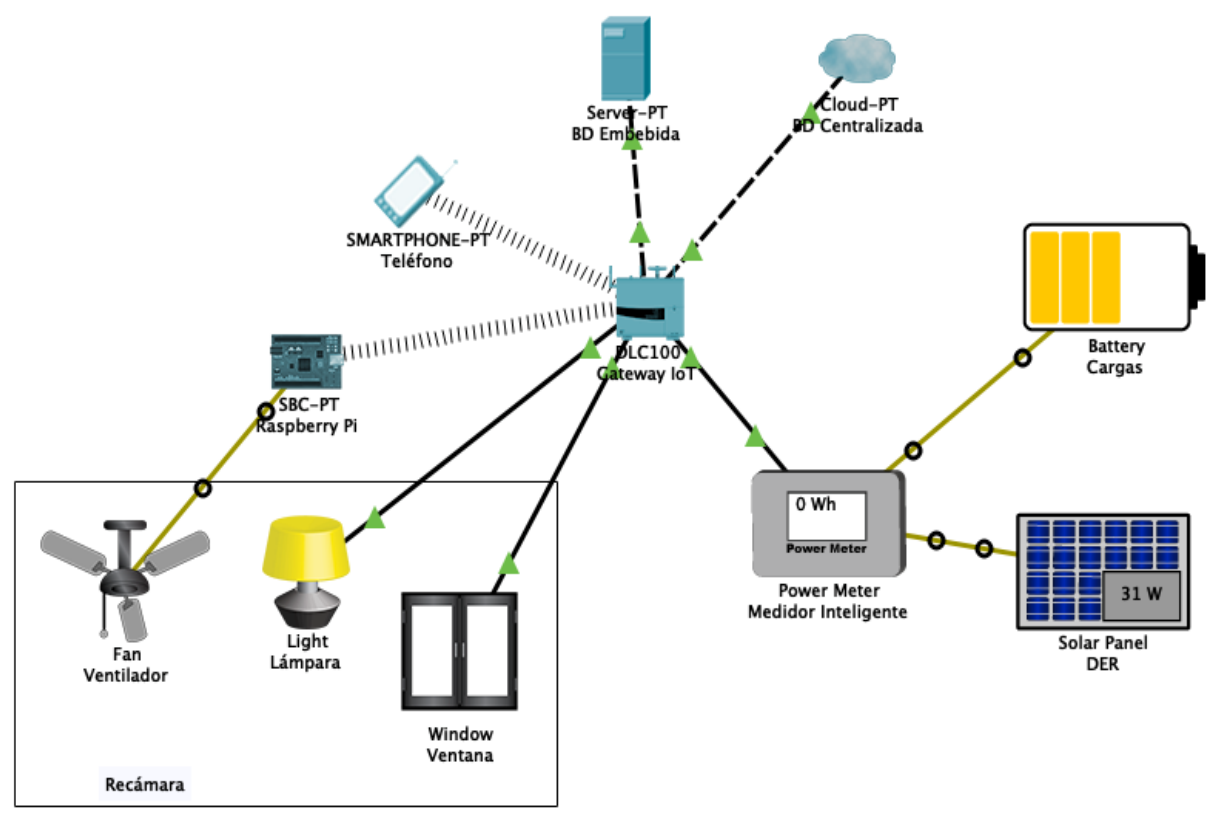

Fig. 1. Topología de simulación básica de un hogar inteligente.

Otra de las limitantes del simulador es el contar con pocas capacidades de almacenamiento de información, ya que, aunque no disponen de base de datos en forma, si se tiene la capacidad de almacenar información en archivos, motivo por el cual el medidor inteligente utiliza un archivo separado por comas (CSV) en donde se guarda la información proveniente de cada dispositivo con la siguiente estructura: timestamp, id_dispositivo, tipo, valor. Tal y como puede observarse en la Tabla 1.

Tabla 1. Ejemplo de Base de Datos del Medidor Inteligente

\begin{tabular}{llll}
\hline timestamp & Id_dispositivo & Tipo & Valor \\
\hline 20190301.00000015 & 0xAB990F & C & 0.06 \\
20190301.00000022 & 0xBB4312 & P & 1 \\
20190301.00000037 & 0xBBCD23 & C & 0.14 \\
20190301.00000049 & 0x1468BC & C & 0.001 \\
20190301.00000103 & 0x29FA1C & C & 0.12 \\
\hline
\end{tabular}

En la Tabla 1 se puede observar que el tipo C corresponde con el consumo de energía y $\mathrm{P}$ a la producción. Los valores están dados en $\mathrm{Kw} / \mathrm{h}$, mientras que los IDs de los dispositivos de la simulación son: 0xAB990F para lámpara, 0xBB4312 para panel fotovoltaico, 0xBBCD23 para la batería, 0x1468BC para la ventana inteligente y 0x29FA1C para el ventilador.

La funcionalidad de almacenamiento también puede integrarse a los dispositivos de IoT permitiendo tener una pequeña base de datos empotrada en cada uno de ellos. 
Juan C. Olivares-Rojas, Enrique Reyes-Archundia, José A. Gutiérrez-Gnecchi, et al.

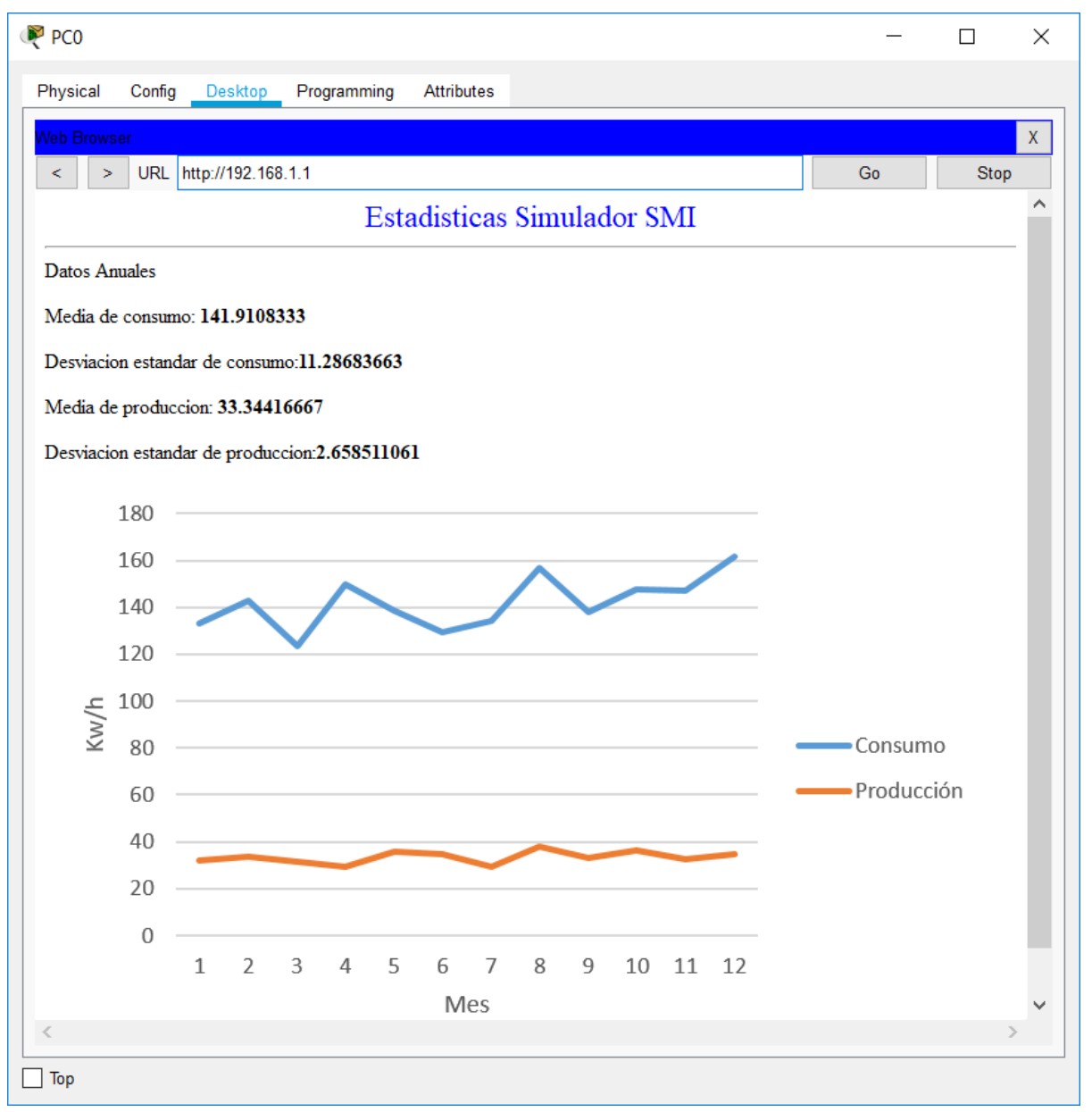

Fig. 2. Resultados de la simulación en forma anual.

Por otra parte, los dispositivos de producción de energía no toman en cuenta otras variables de la señal eléctrica importante en las simulaciones como son la frecuencia y el voltaje; motivo por el cual fue agregado esta variante al panel fotovoltaico y áerogenerador para tener estos datos.

Se agregó un dispositivo raspberry pi para simular un Concentrador de Datos (CD) dentro de la arquitectura AMI. Un CD permite almacenar las lecturas de diversos medidores inteligentes y reportarlos a los servidores de la empresa eléctrica.

La funcionalidad de monitoreo de energía implementada en el medidor inteligente también fue colocado en el CD y en el dispositivo Servidor.

Dentro del medidor inteligente se agregaron rutinas para el procesamiento estadísticos de los datos de medición implementando medidas de tendencia central. Esta funcionalidad también se migró a los CD y Servidor para brindar una plataforma de procesamiento distribuido edge-fog-cloud computing. 


\section{Resultados}

En el presente trabajo, se realizó la simulación de la red presentada en la sección anterior durante un periodo de 30 días representando un año físico (el software permite adelantar los tiempos de la simulación).

Los datos de los dispositivos de consumo y producción de energía son reportados cada 15 minutos (el cual es el promedio de reporte en AMI) por lo que la información generada fue de un total de 175,200 registros para un tamaño aproximado de 8.75 MB.

Las funciones de analítica de datos fueron ejecutadas por hora, por día y por mes observando que los tiempos de consulta a mayor cantidad de datos son más lentos debido a que el acceso a los registros en el archivo es de manera secuencial.

La Figura 2 muestra los resultados finales de la simulación a un año con datos creados por los dispositivos de consumo/producción modificados.

Aunque el simulador trabaja con Python, muchas de las librerías populares para el manejo de datos como numpy, pandas, matplotlib, etc., no están disponibles en el simulador PacketTracert.

Se pueden reutilizar módulos desarrollados en otros proyectos, por lo que es posible utilizar la implementación de algoritmos de aprendizaje máquina sencillos como clasificadores y técnicas de agrupamiento.

\section{Conclusiones y trabajo futuro}

En este trabajo se demuestra que es posible realizar simulaciones de SMI de una manera integral considerando no solo elementos de energía eléctrica sino además considerando factores como la comunicación, ciber seguridad y minería de datos.

Aunque la simulación presenta buenos resultados aún falta realizar muchas tareas, muchas de las cuales se tienen que realizar desde cero, lo cual ha implicado invertir demasiado tiempo de desarrollo.

Además, se puede observar que los datos empiezan a crecer en una manera considerable particularmente en los CD y sobre todo en los Servidores.

Como trabajos futuros se encuentran: extender la simulación a múltiples HAN, medidores inteligentes y sobre todo a la implementación de algoritmos más robustos para la predicción de datos como series de tiempo. A su vez se pretende realizar ciberataques para medir el grado de confidencialidad, integridad y disponibilidad de los datos simulados.

Por otra parte, se tiene contemplado integrar funcionalidad de micropython y llevar el trabajo de simulación sobre medidores inteligentes realizados con placas como Raspberry Pi.

Agradecimientos. Este trabajo está parcialmente financiado por el Tecnológico Nacional de México dentro del proyecto con registro 6385.19-P. 


\section{Referencias}

1. Morello, R., De Capua, C., Fulco, G., Mukhopadhyay, S.C.: A smart power meter to monitor energy flow in smart grids: The role of advanced sensing and IOT in the electric grid of the future. IEEE Sensors Journal (2017)

2. Alahakoon, D., Yu, X.: Smart Electricity Meter Data Intelligence for Future Energy Systems: A Survey. IEEE Transactions on Industrial Informatics (2016)

3. Wang, Y., Chen, Q., Kang, C., Xia, Q., Luo, M.: Sparse and Redundant Representation-Based Smart Meter Data Compression and Pattern Extraction. IEEE Transactions on Power Systems (2017)

4. Cooper Power Systems- Eaton: CYME Power Engineering Software. Disponible en: http://www.cyme.com/, último acceso: 2019/05/11

5. DigSILENT: PowerFactory. Disponible en: https://www.digsilent.de/en/powerfactory.html, último acceso: 2019/05/11

6. Neplan: Neplan Smarter Tools. Disponible en: https://www.neplan.ch/, último acceso: 2019/05/11

7. PowerTech Labs: DSATools. Disponible en: https://www.powertechlabs.com/dsatoolsservices/, último acceso: 2019/05/11

8. Electrical Power System Analysis \& Operation Software: etap Powering Success. Disponible en: https://etap.com/18, último acceso: 2019/05/11

9. SKM System Analysis, Inc.: SKM PowerTools. Disponible en: http://skm.com/, último acceso: 2019/05/11

10. PowerWorld Corporation: PowerWorld Simulator. Disponible en: https://www.powerworld.com/products/simulator/overview, último acceso: 2019/05/11

11. EasyPower: EasyPower Power System Software. Disponible en: https://www.easypower.com/, último acceso: 2019/05/11

12. Manitoba Hydro International Ltd.: PSCAD/EMTC. Disponible en: https://hvdc.ca/pscad/, último acceso: 2019/05/11

13. Orcad: Orcad Professional PCB Design. Disponible en: https://www.orcad.com/, último acceso: 2019/05/11

14. CircuitLab: CircuitLab. Disponible en: https://www.circuitlab.com/, último acceso: 2019/05/11

15. MathWorks: Matlab. Disponible en: https://la.mathworks.com/products/matlab.html, último acceso: 2019/05/11

16. MathWorks: Simulink. Disponible en: https://la.mathworks.com/products/simulink.html, último acceso: 2019/05/11

17. MathWorks: RTL-SDR Support from Communications System Toolbox. Disponible en: https://www.mathworks.com/hardware-support/rtl-sdr.html, último acceso: 2019/05/ 11

18. MathWorks: MATLAB and Simulink for IoT Applications. Disponible en: https://la.mathworks.com/solutions/internet-of-things.html, último acceso: 2019/05/11

19. MathWorks: MATLAB para Comunicaciones Inlámbricas. Disponible en: https://la.mathworks.com/solutions/wireless-communications.html, último acceso: 2019/05/11

20. MathWorks: Digital Signal Processing (DSP). Disponible en: https://la.mathworks.com/solutions/dsp.html, último acceso: 2019/05/11

21. Shlebik, T., et al.: The Development of a Simulation-Based Smart Grid Communication Management System Using MATLAB. In: 2017 International Conference on Green Energy Conversion Systems (GECS). Hammamet, Tunisia. 23-25 de marzo (2017)

22. Pochacker, M., et al.: Simulating the Smart Grid. In: IEEE PowerTech 2013 Institute of Networked and Embedded Systems/ Lakeside Labs Alpen-Adria Universitat Klangenfurt, Austria (2013) 
23. RTDS Technologies: Real Time Digital Power Systems. Disponible en: https://www.rtds.com/, último acceso: 2019/05/11

24. OPAL-RT Technologies: OPAL RT. Disponible en: https://www.opal-rt.com/, último acceso: 2019/05/11

25. OPAL-RT Technologies: RT-LAB. Disponible en: https://www.opal-rt.com/software-rt-lab/, último acceso: 2019/05/11

26. Cisco: Cisco Packet Tracer. Disponible en: https://www.cisco.com/c/dam/en_us/trainingevents/netacad/course_catalog/docs/Cisco_PacketTracer_DS.pdf, último acceso: 2019/05/11

27. Delamare, J., Bitachon, B., Peng, Z., Wang, Y., Haverkort, B.R., Jongerden, M.R.: Development of a Smart Grid Simulation Environment. Electronic Notes in Theoretical Computer Science, vol. 318, pp. 19-29 (2015)

28. Pang, C., Vyatkin, V., Deng, Y., Sorouri, M.: Virtual smart metering in automation and simulation of energy-efficient lighting system. In: 2013 IEEE 18th Conference on Emerging Technologies \& Factory Automation (ETFA), Cagliari, pp. 1-8 (2013)

29. Zhang, T., Nuttall, W.J.: An Agent Based Simulation of Smart Metering Technology Adoption. doi: https://doi.org/10.17863/CAM.5130 (2007)

30. Li, W., Zhang, X.: Simulation of the smart grid communications: Challenges, techniques, and future trends. Computers \& Electrical Engineering 40(1), 270-288 (2014)

31. Sahu, A., Goulart, A., Butler-Purry, K.: Modeling AMI network for real-time simulation in NS-3. In: Principles, Systems and Applications of IP Telecommunications (IPTComm), Chicago, IL, pp. 1-8 (2016) 